\title{
Gene Expression Profiling and Assessment of Vitamin D and Serotonin Pathway Variations in Patients With Irritable Bowel Syndrome
}

\author{
Christopher M Dussik, ${ }^{1}$ Maryam Hockley, ${ }_{1}$ Aleksandra Grozić, ${ }^{1}$ Ichiro Kaneko, ${ }^{1,2}$ Lin Zhang, ${ }^{1}$ Marya S Sabir, ${ }^{1}$ Jin Park, ${ }^{3}$ Jie Wang, ${ }^{3}$ \\ Cheryl A Nickerson, ${ }^{3}$ Steven H Yale, ${ }^{4}$ Christopher J Rall, ${ }^{5}$ Amy E Foxx-Orenstein, ${ }^{6}$ Connie M Borror, ${ }^{1 \dagger}$ Todd R Sandrin, ${ }^{1}$ and \\ Peter W Jurutka ${ }^{1,2 *}$ \\ ${ }^{1}$ School of Mathematical and Natural Sciences, Arizona State University, Phoenix, AZ, USA; ${ }^{2}$ Department of Basic Medical Sciences, University \\ of Arizona College of Medicine, Phoenix, AZ, USA; ${ }^{3}$ School of Life Sciences, Biodesign Institute, Arizona State University, Tempe, AZ, USA; \\ ${ }^{4}$ Department of Medicine, North Florida Regional Medical Center, Gainesville, FL, USA; ${ }^{5}$ Department of Internal Medicine, Marshfield Clinic, \\ Marshfield, WI, USA; and ${ }^{6}$ Department of Gastroenterology and Hepatology, Mayo Clinic College of Medicine, Scottsdale, AZ, USA
}

\begin{abstract}
Background/Aims
Irritable bowel syndrome (IBS) is a multifaceted disorder that afflicts millions of individuals worldwide. IBS is currently diagnosed based on the presence/duration of symptoms and systematic exclusion of other conditions. A more direct manner to identify lBS is needed to reduce healthcare costs and the time required for accurate diagnosis. The overarching objective of this work is to identify gene expression-based biological signatures and biomarkers of IBS.
\end{abstract}

\section{Methods}

Gene transcripts from 24 tissue biopsy samples were hybridized to microarrays for gene expression profiling. A combination of multiple statistical analyses was utilized to narrow the raw microarray data to the top 200 differentially expressed genes between IBS versus control subjects. In addition, quantitative polymerase chain reaction was employed for validation of the DNA microarray data. Gene ontology/pathway enrichment analysis was performed to investigate gene expression patterns in biochemical pathways. Finally, since vitamin $D$ has been shown to modulate serotonin production in some models, the relationship between serum vitamin $D$ and IBS was investigated via 25-hydroxyvitamin D (25[OH]D) chemiluminescence immunoassay.

\section{Results}

A total of 858 genetic features were identified with differential expression levels between IBS and asymptomatic populations. Gene ontology enrichment analysis revealed the serotonergic pathway as most prevalent among the differentially expressed genes. Further analysis via real-time polymerase chain reaction suggested that IBS patient-derived RNA exhibited lower levels of tryptophan hydroxylase-1 expression, the enzyme that catalyzes the rate-limiting step in serotonin biosynthesis. Finally, mean values for $25(\mathrm{OH}) \mathrm{D}$ were lower in IBS patients relative to non-IBS controls.

\section{Conclusions}

Values for serum 25(OH)D concentrations exhibited a trend towards lower vitamin D levels within the IBS cohort. In addition, the expression of select IBS genetic biomarkers, including tryptophan hydroxylase 1, was modulated by vitamin D. Strikingly, the direction of gene regulation elicited by vitamin D in colonic cells is "opposite" to the gene expression profile observed in IBS patients, suggesting that vitamin D may help "reverse" the pathological direction of biomarker gene expression in IBS. Thus, our results intimate that IBS pathogenesis and pathophysiology may involve dysregulated serotonin production and/or vitamin D insufficiency.

(J Neurogastroenterol Motil 2018;24:96-106)

\section{Key Words}

25-Hydroxyvitamin D; Biomarkers; DNA Microarray; Serotonin; Tryptophan hydroxylase

Received: February 16, 2017 Revised: August 3, 2017 Accepted: August 16, 2017

(a) This is an Open Access article distributed under the terms of the Creative Commons Attribution Non-Commercial License (http://creativecommons. org/licenses/by-nc/4.0) which permits unrestricted non-commercial use, distribution, and reproduction in any medium, provided the original work is properly cited.

${ }^{*}$ Correspondence: Peter W Jurutka, PhD School of Mathematical and Natural Sciences, 4701 W. Thunderbird Rd., Glendale, AZ 85306, USA

${ }^{\dagger}$ Deceased. Tel: +1-602-543-6087, Fax: +1-602-543-6087, E-mail: pjurutka@asu.edu 


\section{Introduction}

Irritable bowel syndrome (IBS) is one of the most common gastrointestinal (GI) disorders afflicting between $10-15 \%$ of the global population. ${ }^{1}$ Primary symptoms associated with IBS include abdominal pain and irregular bowel movements that, based upon the predominant symptom pattern, may be utilized to subdivide the syndrome into 3 classifications: diarrhea-predominant IBS (IBS-D), constipation-predominant IBS (IBS-C), and mixed IBS (IBS-M). ${ }^{2}$ However, the subtype of IBS that an individual is classified does not necessarily remain static; patients can evolve over time from diarrheic or mixed type to constipative. The chronic and erratic nature of IBS can lead to a multitude of secondary effects, such as depression, anxiety, poor quality of life, insomnia, and sexual dysfunction. ${ }^{3}$ Using the current diagnostic model for IBS, the Rome IV criteria, symptoms must be present for 3 months or longer before diagnoses can be established. ${ }^{4}$ Other disorders with IBS-like symptoms that should be excluded include celiac disease, inflammatory bowel disease (IBD), malabsorptive disorders, pelvic floor disorders, and colon cancer. Further, once diagnosed, treatment for IBS patients may be mostly palliative in nature with dietary changes and pharmacological agents to alleviate symptoms in some patients. ${ }^{5}$ The chronic symptoms, costly process, and lack of a fully satisfactory or curative treatment contribute to a significantly decreased quality of life for those affected.

Despite the prevalence of IBS and the disease burden it places on individual patients as well as the healthcare system, the pathophysiology of the disorder remains enigmatic, with some exceptions. Results from independent familial and twin studies suggest that IBS may have a genetic etiology. ${ }^{6}$ These findings have led to significant interest in genetic characteristics that may be leveraged to diagnose IBS. For example, this approach has exhibited success in identifying specific alleles in 2 of the most prevalent forms of IBD, namely Crohn's disease (CD) and ulcerative colitis (UC). ${ }^{7}$ Genetic polymorphisms have also been discovered that may be associated with IBS; thus IBS appears to be a complex and multifactorial disorder with possible genetic components similar to $\mathrm{CD}$ and $\mathrm{UC}$.

DNA microarrays have been used to study gene expression in colonic tissue samples from IBD, UC, and CD patients. ${ }^{9-11}$ DNA microarrays have also been employed to characterize gene expression in IBS, ${ }^{12-14}$ albeit somewhat less completely. Based on genes identified via microarray results, and predictive analysis of microarrays evaluation, a test utilizing a series of 32 probes representing differentially expressed genes was constructed that purportedly afforded 70\% accuracy in distinguishing between mucosal tissues derived from IBS and non-IBS individuals. ${ }^{14}$ Identification of additional differentially expressed genes may lead to development of a modified assay with higher accuracy, and further insight into the molecular foundation of IBS. Thus, a test to probe expression of IBS-relevant genes, as well as other biomarkers, may afford better discrimination between symptomatic and asymptomatic individuals, and is highly desirable. .11,13,14 $^{-1}$

Irregularities in serotonergic metabolism have also been hypothesized as potential causal factors for IBS. ${ }^{15}$ Although commonly associated with central nervous function, the majority of serotonin (95\%) is synthesized within the GI tract. ${ }^{16}$ Of this serotonin, over $90 \%$ is produced by enterochromaffin cells whereas the remainder is synthesized by neurons within the myenteric plexus. ${ }^{17}$ Two genes significantly involved in serotonin metabolism display decreased levels of expression in IBS: the serotonin transporter and the synthetic rate-limiting enzyme, tryptophan hydroxylase 1 (TPH1). ${ }^{18}$ Other studies also point to disordered enteric serotonergic signaling in the pathophysiology of IBS, ${ }^{19,20}$ although a clear mechanism has yet to emerge. Nonetheless, a serotonergic model for IBS has shown some efficacy in the design of novel therapeutic regimens for IBS. Selective serotonin reuptake inhibitors are serotonin transporter inhibitors originally designed as antidepressants which have also exhibited potential to alleviate IBS symptoms. ${ }^{21}$

Upstream regulation of serotoninergic metabolic processes may also be involved in IBS pathogenesis. For example, while commonly associated with its role in $\mathrm{Ca}^{2+} / \mathrm{PO}_{4}^{3-}$ homeostasis, vitamin $\mathrm{D}$ is crucial in the modulation of a variety of other pathways, including those involved in anti-inflammatory and immunomodulatory activity. ${ }^{22}$ Thus, vitamin $\mathrm{D}$ has been proposed as a potential treatment for $\mathrm{IBS}^{23}$ as well as IBD. ${ }^{24}$ Moreover, in a recent report, the production of the rate-limiting enzyme in serotonin production, TPH, was significantly stimulated by the active hormonal vitamin D metabolite, 1,25-dihydroxyvitamin $\mathrm{D}(1,25 \mathrm{D})$, in serotonergic neurons. ${ }^{25}$

If IBS is linked to an imbalance in serotonin production, vitamin D could play a role in IBS pathogenesis and may provide novel insights into new modes of IBS management. Further, given the previously-discussed possible genetic etiology of IBS, we postulated that the vitamin $\mathrm{D}$ profile and individual transcriptome patterns from our patient cohort could be explored in an attempt to identify a more efficacious and cost-effective diagnostic model for IBS. Thus, this study analyzed physiological levels of vitamin D and the transcriptional pattern of serotonergic gene expression within IBS and control patient populations and identified other genes as potential novel IBS biomarkers. 


\section{Materials and Methods}

\section{Sample Acquisition and Storage}

A total of 29 patients consisting of 28 non-Hispanic Caucasians and 1 Asian participant were recruited over the course of this study. Mucosal tissue samples were obtained from 24 subjects as described by Costello et al. ${ }^{10}$ These samples included individuals suffering from untreated IBS and individuals that did not display IBS symptoms (which served as the experimental control). Samples were obtained from the sigmoid colon $(25 \mathrm{~cm}$ measured from withdrawal). All research was approved by the Arizona State University IRB (No. 1106006538) and the Marshfield Clinic Research Foundation IRB (No. YAL10308) and all subjects were consented using a written informed consent form that was approved by the IRB. All procedures performed in studies involving human participants were in accordance with the ethical standards of the institutional and/or national research committee and with the 1964 Helsinki declaration and its later amendments or comparable ethical standards.

\section{RNA Isolation: Cell Culture-based}

HCT-116 colorectal cancer cells (male-derived) obtained from the American Type Culture Collection (Manassas, VA, USA) were passaged in DMEM/high glucose media supplemented with $10 \%$ fetal bovine serum and penicillin/streptomycin. Cells were plated at 800000 cells/well in 6 well plates and treated with either $10 \mathrm{nM}$ $1,25 \mathrm{D}$ or ethanol vehicle and incubated in a humidified environment composed of $5 \% \mathrm{CO}_{2}$ at $37^{\circ} \mathrm{C}$ for 24 hours. Total RNA was extracted from each well using the Aurum total RNA mini kit (BioRad, Hercules, CA, USA) following the manufacturer's protocol. Spectroscopy was employed to obtain $\mathrm{A}_{260}$ and $\mathrm{A}_{280}$ values to quantify and assess RNA yield/quality.

\section{RNA Isolation: Tissue-based}

Total RNA was obtained using the Aurum total RNA mini kit (Bio-Rad) and manufacturer's protocol. Tissue samples were resuspended in $700 \mu \mathrm{L}$ of Aurum cell lysis buffer and the cells were lysed via sonication. Spectroscopy was utilized to assess quantity and quality of the RNA. RNA derived from non-IBS control patients were combined into an RNA pool from 8 control samples prior to downstream application.

\section{Microarray Analysis: Tissue-based}

RNA quality and concentration was determined on a Bioanalyzer (Agilent, Santa Clara, CA, USA) and NanoDrop spectrophotometer (ThermoFisher, Waltham, MA, USA). One-color labeling reactions were prepared using the Agilent one-color protocol (version 6.5) with $400 \mathrm{ng}$ total RNA input. Then, $400 \mathrm{ng}$ of total RNA with the appropriate concentration of RNA spike-in controls was converted into double stranded cDNA using an oligo (dT) primer linked to the $\mathrm{T} 7$ promoter sequence and Moloney murine leukemia virus reverse transcriptase. The double stranded cDNA was then

Table 1. Cohort Sample Characteristics

\begin{tabular}{|c|c|c|c|c|c|c|}
\hline $\begin{array}{c}\text { Sample } \\
\text { ID }\end{array}$ & Classification & $\begin{array}{c}\text { IBS } \\
\text { subtype }\end{array}$ & Age & Gender & Race & $\begin{array}{c}\text { Serum } \\
25(\mathrm{OH}) \mathrm{D} \\
(\mathrm{ng} / \mathrm{mL})\end{array}$ \\
\hline IBS-006 & Control & $\mathrm{N} / \mathrm{A}$ & 55 & $\mathrm{~F}$ & Caucasian & 32.7 \\
\hline IBS-010 & Control & $\mathrm{N} / \mathrm{A}$ & 54 & M & Caucasian & 13.8 \\
\hline IBS-012 & Control & $\mathrm{N} / \mathrm{A}$ & 50 & $\mathrm{~F}$ & Caucasian & 30.6 \\
\hline IBS-016 & Control & $\mathrm{N} / \mathrm{A}$ & 52 & $\mathrm{~F}$ & Caucasian & 50.1 \\
\hline IBS-022 & Control & $\mathrm{N} / \mathrm{A}$ & 60 & $\mathrm{~F}$ & Caucasian & 63.6 \\
\hline IBS-026 & Control & $\mathrm{N} / \mathrm{A}$ & 53 & M & Caucasian & 44.8 \\
\hline IBS-027 & Control & $\mathrm{N} / \mathrm{A}$ & 50 & M & Caucasian & 24.9 \\
\hline IBS-056 & Control & $\mathrm{N} / \mathrm{A}$ & 51 & $\mathrm{~F}$ & Asian & 84.6 \\
\hline IBS-017 & Control & $\mathrm{N} / \mathrm{A}$ & 57 & $\mathrm{~F}$ & Caucasian & 31.3 \\
\hline IBS-019 & Control & $\mathrm{N} / \mathrm{A}$ & 50 & M & Caucasian & 47.8 \\
\hline IBS-030 & Control & $\mathrm{N} / \mathrm{A}$ & 47 & M & Caucasian & 51.3 \\
\hline IBS-043 & Control & $\mathrm{N} / \mathrm{A}$ & 54 & $\mathrm{~F}$ & Caucasian & 63.1 \\
\hline IBS-045 & Control & $\mathrm{N} / \mathrm{A}$ & 50 & $\mathrm{~F}$ & Caucasian & 45.2 \\
\hline IBS- $011^{\mathrm{a}}$ & Control & $\mathrm{N} / \mathrm{A}$ & 50 & $\mathrm{~F}$ & Caucasian & 87.2 \\
\hline IBS- $020^{\mathrm{a}}$ & Control & $\mathrm{N} / \mathrm{A}$ & 47 & M & Caucasian & 52.5 \\
\hline IBS-02 $1^{\mathrm{a}}$ & Control & $\mathrm{N} / \mathrm{A}$ & 49 & $\mathrm{~F}$ & Caucasian & 60.3 \\
\hline IBS-032 ${ }^{\mathrm{a}}$ & Control & $\mathrm{N} / \mathrm{A}$ & 50 & $\mathrm{~F}$ & Caucasian & 32.7 \\
\hline IBS- $046^{\mathrm{a}}$ & Control & $\mathrm{N} / \mathrm{A}$ & 51 & $\mathrm{~F}$ & Caucasian & 42.4 \\
\hline IBS-002 & Case & IBS-D & 56 & $\mathrm{~F}$ & Caucasian & $\mathrm{ND}$ \\
\hline IBS-003 & Case & Unknown & 49 & $\mathrm{~F}$ & Caucasian & 48.0 \\
\hline IBS-004 & Case & IBS-M & 51 & $\mathrm{~F}$ & Caucasian & 23.2 \\
\hline IBS-007 & Case & IBS-M & 53 & $\mathrm{~F}$ & Caucasian & 45.5 \\
\hline IBS-009 & Case & IBS-D & 58 & M & Caucasian & 35.8 \\
\hline IBS-013 & Case & IBS-C & 57 & $\mathrm{~F}$ & Caucasian & 81.8 \\
\hline IBS-024 & Case & IBS-M & 51 & M & Caucasian & 60.8 \\
\hline IBS-025 & Case & IBS-D & 55 & M & Caucasian & 25.7 \\
\hline IBS-028 & Case & IBS-D & 55 & $\mathrm{~F}$ & Caucasian & 16.1 \\
\hline IBS-033 & Case & IBS-M & 46 & $\mathrm{~F}$ & Caucasian & 26.1 \\
\hline IBS-034 & Case & IBS-M & 41 & $\mathrm{~F}$ & Caucasian & 46.0 \\
\hline
\end{tabular}

${ }^{a}$ Blood samples only.

IBS, irritable bowel syndrome; 25(OH)D, 25-hydroxyvitamin D; N/A, not applicable (due to control patient status); F, female; M, male; IBS-D, diarrhea-predominant IBS; IBS-M, mixed IBS; IBS-C, constipation-predominant IBS; ND, not determined.

A total of 29 patients were enrolled in this study. 
in vitro transcribed by T7 RNA polymerase, which simultaneously amplifies and incorporates cyanine 3-labeled cytidine triphosphate into the resulting cRNA. Labeled cRNA was then purified using RNeasy columns (Qiagen, Germantown, MD, USA) and the labeling efficiency determined by the NanoDrop spectrophotometer. Next, the labeled cRNA was fragmented and placed on the SurePrint G3 Human GE microarray (Ambry Genetics, Aliso Viejo, CA, USA) and hybridized at $65^{\circ} \mathrm{C}$ for approximately 17 hours. The arrays were washed and scanned at $3 \mu \mathrm{M}$ resolution on an Agilent G2565CA high-resolution scanner. Finally, data processing was performed using Agilent's Feature Extraction software (version 11.01.1) using protocol GE1_1100_Jul11 and the $60 \mathrm{~K}$ grid file 039494 D_F_20120628.

\section{Identification of Differentially Expressed Genes and Gene Ontology/Pathway Enrichment Analysis}

Background-corrected intensities were normalized by the "limma” package ${ }^{26}$ in R. Differentially expressed genes between groups were obtained by combining the lists of features with $P$-values $<$ 0.05 from the "limma" linear model $t$ test, the Mann-Whitney U

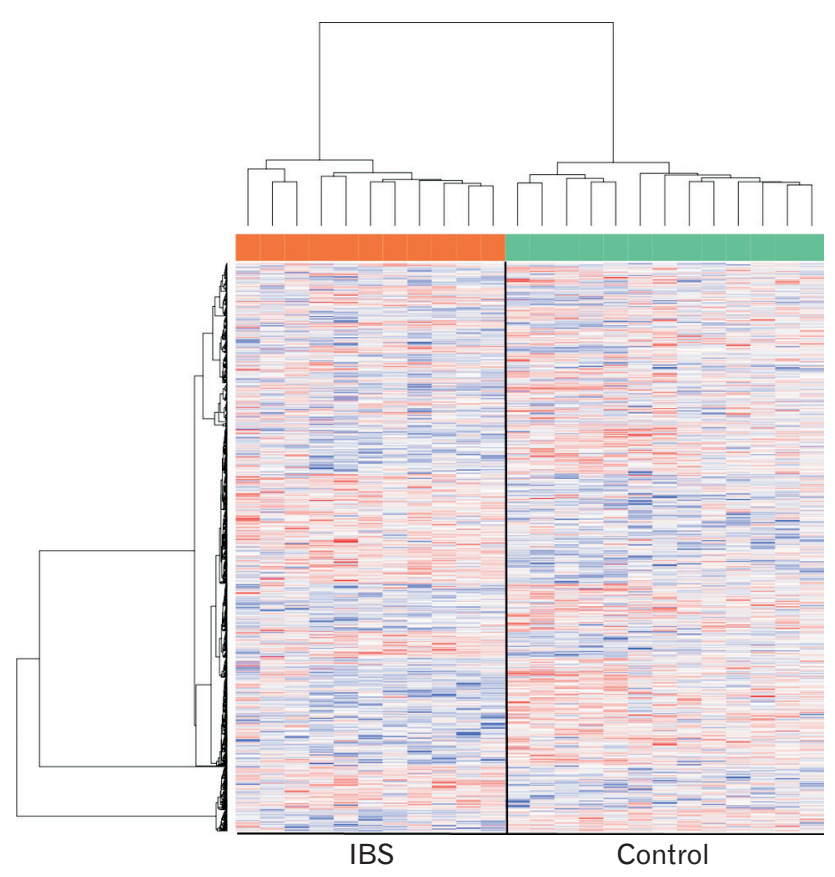

Figure 1. Differential gene expression in irritable bowel syndrome (IBS). A heat map illustrating 858 genetic features with differential expression levels between IBS and asymptomatic populations, with $P$ values $<0.05$ as determined through the Mann-Whitney $U$ test. Color and intensity correlated with the expression patterns for individual biomarkers. Blue is indicative of lower levels of expression while red is indicative of higher levels. test, and the Student's $t$ test. The selected 1564 features were then mapped to genes, and the list of 858 unique genes were subjected to ontology/pathway analysis and visualized by using the "ClueGO"27 and "ReactomeFI" ${ }^{28}$ packages in Cytoscape, ${ }^{29}$ from which enriched terms with $P$-value $<0.05$ were identified.

\section{Quantitative Real-time Polymerase Chain Reaction}

DNase-treated total RNA $(1 \mu \mathrm{g})$ was reverse-transcribed via an iScript cDNA Synthesis kit (Bio-Rad) to prepare $20 \mu \mathrm{L}$ of first strand cDNA. Real-time polymerase chain reaction (PCR) reactions were prepared by adding $1.5 \mu \mathrm{L}$ of cDNA to $5 \mu \mathrm{L}$ FastStart Universal SYBR Green Master Mix (Rox) (Roche Applied Science, Indianapolis, IN, USA) and primers for a total volume of $10 \mu \mathrm{L}$. Reactions were performed in 96 well plates in a BioRad CFX96 thermal cycler using a 40-cycle profile. Data analysis was performed using the comparative $\Delta \Delta \mathrm{Ct}$ method as the means of relative quantitation, normalized to an endogenous reference (GAPDH) and relative to a calibrator (normalized $\mathrm{Ct}$ value from vehicle-treated cells) and expressed as $2^{-\Delta \Delta \mathrm{Ct}}$. The primers utilized during PCR experimentation were as follows:

$\begin{array}{ll}\text { Human GAPDH-forward } & \text { 5'-ACAACTTTGGTATCGTGGAAGGAC-3' } \\ \text { Human GAPDH-reverse } & \text { 5'-CAGGGATGATGTTCTGGAGAG C-3' } \\ \text { Human CYP24A1-forward } & \text { 5'-CAGCGAACTGAACAAATGGTCG-3' } \\ \text { Human CYP24A1-reverse } & \text { 5'-TCTCTTCTCATACAACACGAGGCAG-3' } \\ \text { Human TDRD6-forward } & \text { 5'-AACACCTACACTCTGAAAGCC-3' } \\ \text { Human TDRD6-reverse } & \text { 5'-CGGTTAAATCTCCATCACCTCC-3' } \\ \text { Human MSR1-forward } & \text { 5'-GCTCAATGACAGCTTTGCTTC-3' } \\ \text { Human MSR1-reverse } & \text { 5'-CCAATGAGAGGGATGAGAACTG-3' } \\ \text { Human EPHA3-forward } & \text { 5'-CACCTTTGAGATTGATGCCG-3' } \\ \text { Human EPHA3-reverse } & \text { 5'-GATGCTATTTCTGGAGGTCCG-3' } \\ \text { Human FLT4-forward } & \text { 5'-AGGCATAGACAAGAAAGCGG-3 } \\ \text { Human FLT4-reverse } & \text { 5'-TGTAAAACACCTGGCCTCC-3' } \\ \text { Human ZDHHC15-forward } & \text { 5'-CTGAGGTCCAGAAGCAGATG-3' } \\ \text { Human ZDHHC15-reverse } & \text { 5'-TGGCTTGATCAGATGACACC-3' } \\ \text { Human TPH1-forward } & \text { 5'-TAAGGAGAACAAAGACCATTCC-3' } \\ \text { Human TPH1-reverse } & \text { 5'-TTTCTTCTTTTTGATTTTCGGG-3' }\end{array}$

\section{Analysis of Vitamin D Metabolite Levels}

Measurement of 25-hydroxyvitamin D (25 [OH]D) concentrations from patient serum was performed by Heartland Assays (Ames, IA, USA). A competitive chemiluminescence immunoassay was employed for assessment of $25(\mathrm{OH}) \mathrm{D}$ concentrations, as described previously. ${ }^{30}$ 


\section{Statistical Methods}

Real-time PCR data are reported as means $\pm \mathrm{SD}$. Two-sided Student's $t$ tests were used to measure statistical differences with $P$-values less than 0.05 considered significant. The $25(\mathrm{OH}) \mathrm{D}$ concentrations from patient serum are reported as means $( \pm \mathrm{SD})$, and a two-sided Student's $t$ test was used to measure statistical differences with $P$-values less than 0.05 considered significant. The competitive chemiluminescence immunoassay employed for assessment of $25(\mathrm{OH}) \mathrm{D}$ concentrations included quality assurance and quality control measures such as a pooled serum sample analyzed with batches of study samples to monitor analytical precision and identify possible laboratory shifts over time, as well as testing duplicates in different batches. The coefficient of variation was less than $7 \%$ for $25(\mathrm{OH}) \mathrm{D}$ analyses. All analyses were conducted in a blinded fashion. Microarray data was processed using Agilent's Feature Extraction software (version 11.01.1). Data generated from each sample were subsequently used to identify differentially expressed genes $(P$ $\leq 0.05$ ). Data were normalized using limma. ${ }^{26}$

\section{Results}

\section{Evaluation of Potential Irritable Bowel Syndrome Biomarkers}

Samples contained one cohort of 18 asymptomatic individuals and another group of 11 patients who had been diagnosed with IBS using the Rome III criteria. Information regarding patient age, sex, IBS status, subtype, and serum $25(\mathrm{OH}) \mathrm{D}$ levels is summarized in Table 1. The trimmed mean for $25(\mathrm{OH}) \mathrm{D}$ concentration (lowest/ highest value excepted) in all IBS patients was $38.9 \mathrm{ng} / \mathrm{mL}$ versus $47.4 \mathrm{ng} / \mathrm{mL}$ in control subjects, with a median value of $40.7 \mathrm{ng} / \mathrm{mL}$ in all IBS patients and $46.5 \mathrm{ng} / \mathrm{mL}$ in the control cohort. Moreover, the mean 25(OH)D level in the IBS-D subtype was $25.9 \mathrm{ng} /$ $\mathrm{mL}$ versus $47.7 \mathrm{ng} / \mathrm{mL}$ in all control patients $(P<0.03)$. These data from the current pool of IBS/control patients revealed a trend towards lower vitamin D levels within the IBS population, and perhaps specifically in IBS-D patients.

DNA microarray analyses were performed using colonic RNA

Table 2. Top 30 Enriched Gene Ontology Terms

\begin{tabular}{|c|c|c|c|}
\hline UNIQUE_ID & GO Term & Ontology Source & Term $P$-value \\
\hline GO:0051610 & serotonin uptake & GO_BiologicalProcess_20.03.2014_19h52 & $2.63 \mathrm{E}-04$ \\
\hline GO:0060633 & negative regulation of transcription initiation from RNA polymerasell promoter & GO_BiologicalProcess_20.03.2014_19h52 & $9.12 \mathrm{E}-04$ \\
\hline GO:2000143 & negative regulation of DNA-templated transcription, initiation & GO_BiologicalProcess_20.03.2014_19h52 & 0.002682196 \\
\hline REACTOME:19411 & Type II Na+/Pi cotransporters & REACTOME_21.03.2014 & 0.002682196 \\
\hline GO:0006772 & thiamine metabolic process & GO_BiologicalProcess_20.03.2014_19h52 & 0.005256933 \\
\hline GO:0014063 & negative regulation of serotonin secretion & GO_BiologicalProcess_20.03.2014_19h52 & 0.005256933 \\
\hline GO:0019262 & $\mathrm{N}$-acetylneuraminate catabolic process & GO_BiologicalProcess_20.03.2014_19h52 & 0.005256933 \\
\hline GO:0022824 & transmitter-gated ion channel activity & GO_MolecularFunction_20.03.2014_19h52 & 0.005256933 \\
\hline GO:0022835 & transmitter-gated channelactivity & GO_MolecularFunction_20.03.2014_19h52 & 0.005256933 \\
\hline GO:0034056 & estrogen response element binding & GO_MolecularFunction_20.03.2014_19h52 & 0.005256933 \\
\hline GO:0034154 & toll-like receptor 7 signaling pathway & GO_ImmuneSystemProcess_20.03.2014_19h52 & 0.005256933 \\
\hline GO:0051581 & negative regulation of neurotransmitter uptake & GO_BiologicalProcess_20.03.2014_19h52 & 0.005256933 \\
\hline GO:0051611 & regulation of serotonin uptake & GO_BiologicalProcess_20.03.2014_19h52 & 0.005256933 \\
\hline GO:0051612 & negative regulation of serotonin uptake & GO_BiologicalProcess_20.03.2014_19h52 & 0.005256933 \\
\hline GO:0086015 & SA node cell action potential & GO_BiologicalProcess_20.03.2014_19h52 & 0.005256933 \\
\hline GO:0086018 & SA node cell to atrial cardiac muscle cell signalling & GO_BiologicalProcess_20.03.2014_19h52 & 0.005256933 \\
\hline GO:2000467 & positive regulation of glycogen (starch) synthaseactivity & GO_BiologicalProcess_20.03.2014_19h52 & 0.005256933 \\
\hline REACTOME: 15551 & Catecholaminebiosynthesis & REACTOME_21.03.2014 & 0.005256933 \\
\hline GO:0000711 & meiotic DNA repair synthesis & GO_BiologicalProcess_20.03.2014_19h52 & 0.030233082 \\
\hline GO:0001587 & Gq/11-coupled serotonin receptor activity & GO_MolecularFunction_20.03.2014_19h52 & 0.030233082 \\
\hline GO:0003026 & regulation of systemic arterial blood pressure by aortic arch baroreceptor feedback & GO_BiologicalProcess_20.03.2014_19h52 & 0.030233082 \\
\hline GO:0003294 & atrial ventricular junction remodeling & GO_BiologicalProcess_20.03.2014_19h52 & 0.030233082 \\
\hline GO:0004964 & luteinizing hormone receptor activity & GO_MolecularFunction_20.03.2014_19h52 & 0.030233082 \\
\hline GO:0005335 & serotonin:sodium symporter activity & GO_MolecularFunction_20.03.2014_19h52 & 0.030233082 \\
\hline GO:0005757 & mitochondrial permeability transition pore complex & GO_CellularComponent_20.03.2014_19h52 & 0.030233082 \\
\hline GO:0006585 & dopamine biosynthetic process from tyrosine & GO_BiologicalProcess_20.03.2014_19h52 & 0.030233082 \\
\hline GO:0010607 & negative regulation of cytoplasmic mRNA processing body assembly & GO_BiologicalProcess_20.03.2014_19h52 & 0.030233082 \\
\hline GO:0010610 & regulation of mRNA stability involved in response to stress & GO_BiologicalProcess_20.03.2014_19h52 & 0.030233082 \\
\hline GO:0010747 & positive regulation of plasma membrane long-chain fatty acid transport & GO_BiologicalProcess_20.03.2014_19h52 & 0.030233082 \\
\hline GO:0010904 & regulation of UDP-glucosecatabolic process & GO_BiologicalProcess_20.03.2014_19h52 & 0.030233082 \\
\hline
\end{tabular}

$\mathrm{GO}$, gene ontology.

Data are organized in order of increasing $P$-value. 
derived from IBS and asymptomatic sample populations. Analysis of the raw microarray data established a series of 858 genes/biomarkers that display differential expression (Fig. 1). A tabular list of the top 200 biomarkers exhibiting differential expression between the 2 groups is provided in Supplementary Table 1. This set of genetic features shows considerable functional diversity (eg, folate reception, Golgi-ER transport, lipid-intake, etc) and underscores the complexity of the potential genetic foundation of IBS.

\section{Analysis of Biochemical Pathways Potentially Influenced by Irritable Bowel Syndrome}

To visualize effects of distinct gene expression patterns on biochemical pathways, gene ontology ( $\mathrm{GO}$ ) enrichment analysis was performed on the differentially expressed set of genes, with the top 30 enriched GO terms listed in Table 2. Genes were grouped into pathways in which at least 5 members shared similar interactions (Fig. 2). Of particular interest in the current study are the 2 pathways (Fig. 2A and 2C) that coincide with serotonin metabolism, as these 2 pathways possess the highest number of shared functions of any groups.

\section{Evaluation of Tryptophan Hydroxylase-1 Expression Within the Irritable Bowel Syndrome Cohort}

Due to the prevalence of serotonergic pathways within our GO analysis, we aimed at exploring the potential link between serotonin and IBS in further detail. Quantitative real-time PCR was employed to assess the expression of TPH1 due to its role as the rate-limiting biosynthetic enzyme involved in serotonin production within the enterochromaffin cells of the gut ${ }^{17}$ and its proposed link with IBS pathophysiology. ${ }^{18}$ We compared TPH1 expression in individual gut tissue samples of the IBS cohort to the expression profile obtained from a pooled RNA sample comprised of 8 individuals from the asymptomatic cohort selected at random. The results from this analysis for each IBS cohort sample were plotted against each patient's serum vitamin D concentration (Fig. 3). Of the 11 IBS patients analyzed, 10 samples displayed decreased levels of TPH1 expression relative to the control RNA pool. Additionally, $80 \%$ of the IBS patients displayed both decreased TPH1 expression and $25(\mathrm{OH}) \mathrm{D}$ levels below $50 \mathrm{ng} / \mathrm{mL}$ (the clinical reference range in most laboratories for $25(\mathrm{OH}) \mathrm{D}$ is $20-100 \mathrm{ng} / \mathrm{mL}$, with a preferred range of $30-60 \mathrm{ng} / \mathrm{mL}$ as vitamin $\mathrm{D}$ "sufficient" ${ }^{\text {"1, } 132}$ ).
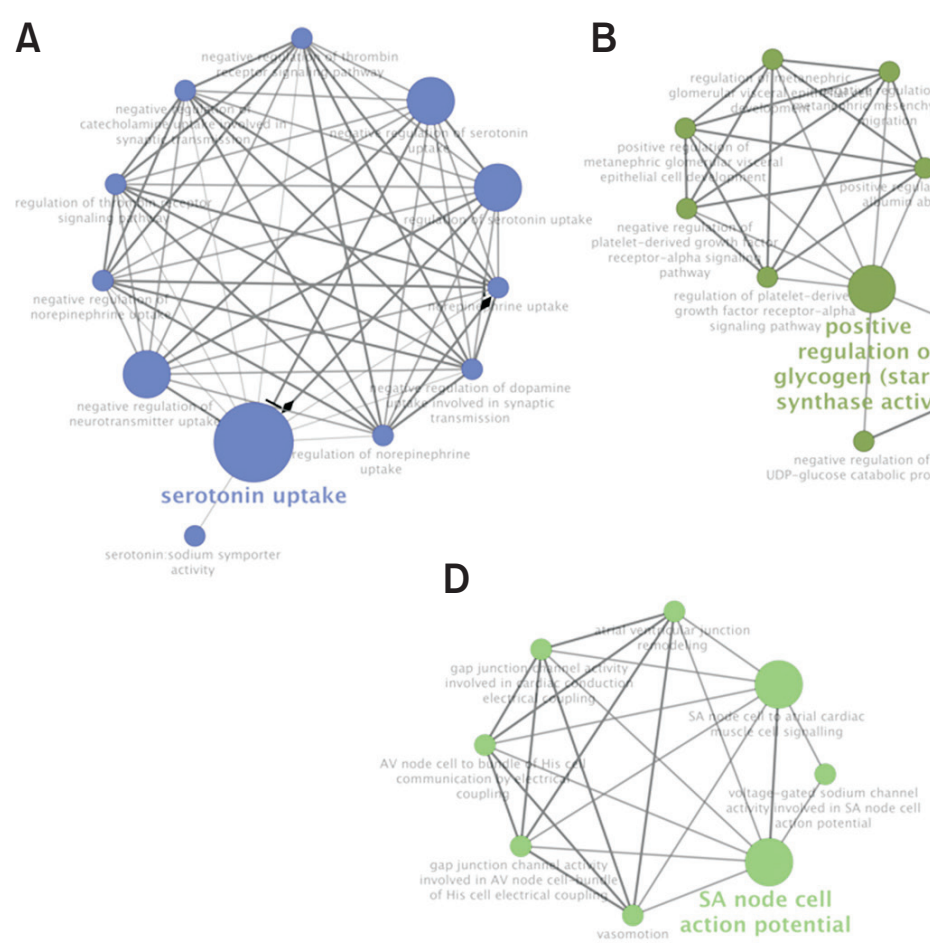

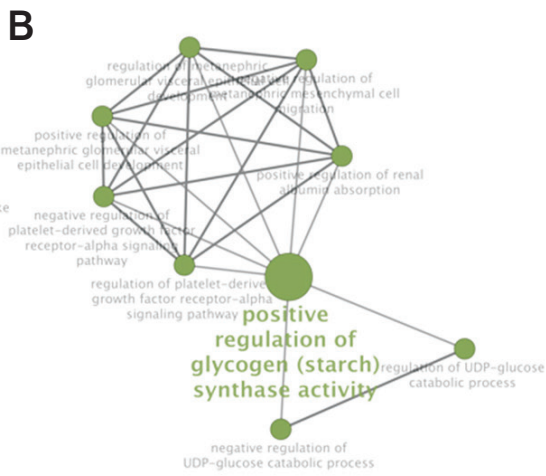

E

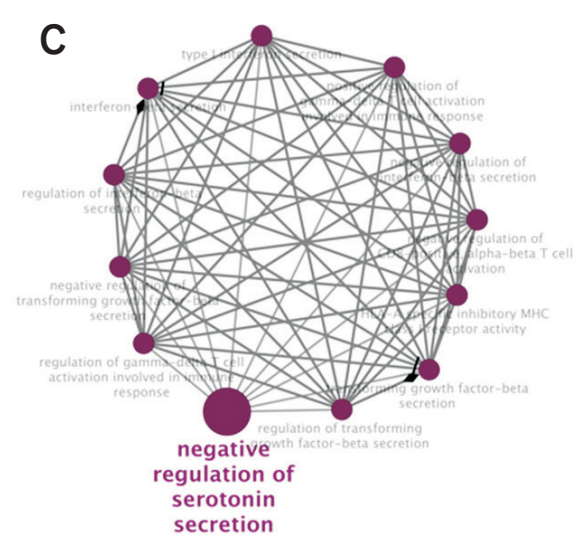

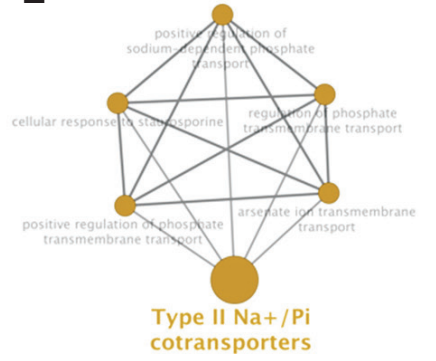

Figure 2. Gene ontology, pathways, and interactions involved with the genes identified to be differentially expressed between the irritable bowel syndrome and the asymptomatic control cohort. (A-E) The interactions shown include only those that possessed 5 or more functions. SA, sinoatrial; $\mathrm{Pi}$, inorganic phosphate. 


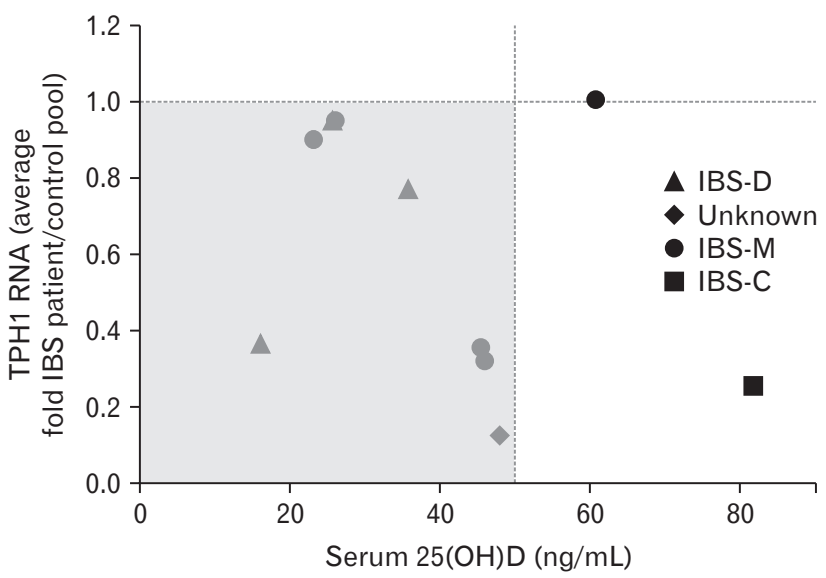

Figure 3. Expression of tryptophan hydroxylase-1 (TPH1) is reduced in irritable bowel syndrome (IBS). Real-time polymerase chain reaction was employed to compare expression patterns observed in IBS patient-derived RNA with expression patterns found in a pooled RNA sample from 8 asymptomatic patients. Fold values were plotted against the sera vitamin D content of each individual IBS patient. The region consisting of values that correlate with both depressed $\mathrm{TPH} 1$ expression and sera vitamin D concentrations below $50 \mathrm{ng} / \mathrm{mL}$ is shaded gray. Note: one IBS patient possessed low (0.27-fold) TPH1 expression but vitamin D levels were not measured, thus this patient is not included in the graph. The data are presented as a ratio of IBS patient:non-IBS control pool to assess the relative gene expression of TPH1 in the IBS cohort. 25(OH)D, 25-hydroxyvitamin D; IBS-D, diarrhea-predominant IBS; IBS-M, mixed IBS; IBS-C, constipationpredominant IBS.

While no clear pattern was observed that could differentiate between the TPH1 expression levels or $25(\mathrm{OH}) \mathrm{D}$ concentrations and IBS subtypes (C, D, or M), the data in Figure 3 (combined with the $25[\mathrm{OH}] \mathrm{D}$ data in Table 1) suggest lower levels of serum vitamin $\mathrm{D}$ and/or colonic TPH1 expression as potential biomarkers for IBS.

\section{Vitamin D Affects the Expression of Several Putative Irritable Bowel Syndrome Biomarkers in Colonic Epithelial Cell-lines}

Colorectal cells (HCT-116) were treated with $10^{-8} \mathrm{M} 1,25 \mathrm{D}$ for 24 hours and the mRNA levels of 5 putative IBS genetic biomarkers were analyzed using real-time PCR (Fig. 4). These genes were selected from an analysis of the DNA microarray data and represent genes with statistically significant, differential regulation in the IBS patients with high fold activation or repression relative to control patients (Supplemental Table 2). Of the 5 IBS biomarker candidate genes tested for regulation by 1,25D, 3 genes (TDRD6, EPHA3, and FLT4) were influenced by the presence of $10^{-8} \mathrm{M}$ vitamin $\mathrm{D}$,

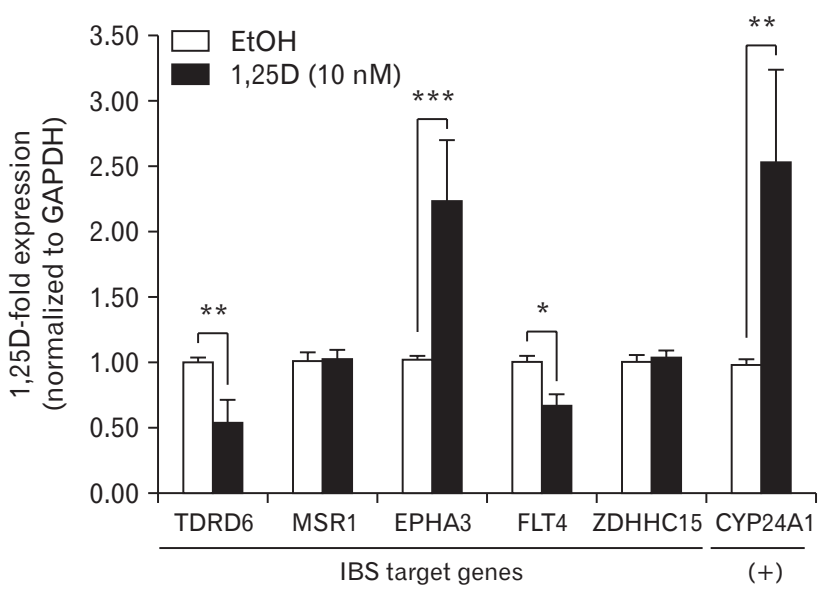

Figure 4. Effect of 1,25-dihydroxyvitamin $\mathrm{D}_{3}(1,25 \mathrm{D})$ on the expression of irritable bowel syndrome (IBS)-candidate gene mRNA in human colonic cells. Cells were treated with $10^{-8} \mathrm{M} 1,25 \mathrm{D}$ for 24 hours, and mRNA levels determined by real-time polymerase chain reaction. Fold induction by $1,25 \mathrm{D}$ is shown on the ordinate for the indicated genes. CYP24A1 $(+)$ is a positive control gene known to be upregulated by vitamin D. Data are representative of at least 3 independent experiments with $3-6$ replicates in each group. ${ }^{*} P<0.05$; ${ }^{*} P<$ $0.005 ;{ }^{* *} P<0.0005$. Preliminary experiments in the noncancerous cell line, HEK-293, reveal similar findings (data not shown). EtOH, ethanol.

while 2 genes (MSR1 and ZDHHC15) were unchanged. Given the role of FLT4 in both angiogenesis and lymphangiogenesis, ${ }^{33}$ the involvement of EPHA3 in axonal migration and pathfinding, ${ }^{34}$ and the hypothesized function of TDRD6 in spliceosome function, ${ }^{35}$ more research is necessary to determine their possible function in IBS pathogenesis. Nonetheless, regardless of the exact mechanism whereby these genes might influence IBS pathophysiology, when tested in response to $1,25 \mathrm{D}$ (Fig. 4), the expression of TDRD6 was repressed 0.54 fold $(P<0.005)$, EPHA3 was induced 2.23 fold $(P$ $<0.0005)$, and FLT4 mRNA was attenuated 0.67 fold $(P<0.05)$. Significantly, DNA microarray data (Supplementary Table 2) indicated that the IBS cohort displayed increased TDRD6 and FLT4 expression and decreased EPHA3 expression. Thus, the direction of gene regulation by $1,25 \mathrm{D}$ in colonic cells is "opposite" to that observed in IBS patients, suggesting that vitamin D treatment may help reverse the pathological direction of biomarker gene expression in IBS.

\section{Discussion}

DNA microarray analysis revealed 858 features that exhibited differential expression between an IBS sample population and as- 
ymptomatic control population. These results provide evidence to support the hypothesis that IBS may be founded upon a genetic or transcriptome basis, as has been suggested previously. ${ }^{14}$ Similar to a previous study, ${ }^{14}$ our list of putative IBS biomarkers (Supplementary Table 1) contains genes that are both up-regulated and downregulated in IBS patients versus healthy controls. We also obtained high concordance between genes identified in the microarray and those subsequently assessed by real-time PCR, similar to the work of Aerssens et al. ${ }^{14}$ However, we observed a higher percentage of IBS up-regulated genes (40\%) than Aerssens et $\mathrm{al}^{14}$ (25\%). Some of these differentially expressed genes may prove useful as screening biomarkers that could be utilized to improve the performance of the Rome IV diagnostic model. We propose that the expression pattern of a subset of these genes may consistently produce a characteristic IBS genetic "fingerprint" (Fig. 1) that could be used as an effective screening tool for rapid diagnosis of IBS-afflicted individuals.

In addition to serving as potential biomarkers, many of the genes located within the differentially expressed gene group may play an etiological role in the IBS pathological state. Analysis of individual gene functions and complex polygenetic interactions is a key first step in discovering a potential causative association between specific gene expression patterns and IBS pathophysiology. Gene ontology interaction analysis was performed to provide a broad overview of our complex microarray dataset. Our analysis revealed that genes involved in serotonin metabolism were well represented in the differentially expressed gene pool. An IBS disease model in which an imbalance of serotonin production or responsiveness may play a key role in IBS pathogenesis has been proposed, and it is possible that defective serotonin signaling is a common feature in the pathogenesis of functional bowel diseases. Pharmacological intervention strategies with serotonergic agents have been successfully employed in some patients as treatment modalities. ${ }^{36}$ Real-time PCR analysis of the rate-limiting enzyme (TPH1) in gut serotonin synthesis was performed to determine whether IBS patients exhibited a decreased store of GI serotonin. This analysis revealed that 10 of the 11 IBS patients tested exhibited decreased levels of TPH1 relative to a non-IBS sample population, although another study ${ }^{37}$ found a statistically insignificant trend towards elevated TPH1 expression in IBS patients.

The active hormonal metabolite of vitamin $\mathrm{D}$ has been shown to modulate serotonin production within neural ${ }^{25}$ and colonic tissue (data not shown). In fact, vitamin D has been proposed as a potential treatment for IBS. ${ }^{38,39}$ Due to the relative abundance of vitamin D responsive elements (VDREs) in hundreds of vitamin D regulated genes throughout the genome, ${ }^{40}$ we postulated that expression of some IBS candidate biomarker genes may be influenced by the presence of the active hormonal metabolite of vitamin $\mathrm{D}, 1,25$-dihydroxyvitamin $\mathrm{D}_{3}(1,25 \mathrm{D}) .^{41}$ The manner in which this potential regulation occurs and the specific genes involved in IBS pathophysiology may suggest possible new approaches to treatment.

We recently identified functional VDREs in the human TPH1 gene. $^{25}$ Taking this into account and considering the potential linkage between IBS and abnormal serotonin metabolism, we examined whether IBS patients exhibited lower serum levels of vitamin D. Nine of the eleven patient samples that were tested displayed 25(OH)D levels below $50 \mathrm{ng} / \mathrm{mL}$ (the range of vitamin D "sufficiency" has been previously defined as $30-60 \mathrm{ng} / \mathrm{mL}$, with the most optimal levels $\geq 50 \mathrm{ng} / \mathrm{mL}^{32}$ ). However, given our small sample size, this finding could have been affected by individual variance in sun-exposure, supplement intake, and overall body fat composition. Nonetheless, serum 25(OH)D levels are recognized as a reliable biomarker of human vitamin D status, and reflect the ability of tissues to synthesize the active hormonal metabolite of vitamin D, $1,25 \mathrm{D}$ which regulates gene expression via the vitamin $\mathrm{D}$ receptor.

In addition, given that vitamin $\mathrm{D}$ regulates the expression of a vast array of genes, ${ }^{42}$ we postulated that lower vitamin D levels may explain some of the differential expression patterns observed in our DNA microarray analyses. To test this hypothesis, HCT116 colonic cells were treated with $10^{-8} \mathrm{M} 1,25 \mathrm{D}$, and real-time PCR was used to analyze the response of 5 different putative IBS biomarkers (TDRD6, MSR1, EPHA3, FLT4, and ZDHC115) which were selected via a combination of DNA microarray analysis and real-time PCR (Supplementary Table 2). The results of this analysis revealed that $1,25 \mathrm{D}$ regulates a subset of potential IBS genes (TDRD6, EPHA3, and FLT4) in a manner that offsets the altered expression patterns within our IBS patient cohort (Fig. 4). This suggests that vitamin D may help ameliorate IBS symptoms by reversing the pathological gene expression of IBS biomarkers.

Serotonin release by enterochromaffin cells embedded within the GI mucosa is used to modulate the strength of smooth muscle contraction and, in turn, GI motility. ${ }^{43}$ High levels of serotonin release correlate with high levels of GI motility, and low levels of serotonin release correlate with low levels of GI motility. ${ }^{44,45}$ Conventionally, IBS-C would be associated with depressed serotonin production, IBS-M with intermediate or alternating serotonin production, and IBS-D with elevated serotonin production. However, 10 of the 11 IBS patient tissue samples analyzed in our cohort exhibited depressed levels of TPH1 expression relative to a sample population of control non-IBS RNA. Previously, we have measured the stimulation of serotonin production in response to vitamin D 
within the serotonergic cell line, RN46A-B14. ${ }^{46}$ Thus, given that TPH1 mRNA was induced by $1,25 \mathrm{D}$ in the gut, it is likely that serotonin is produced in the colon in response to vitamin $\mathrm{D}$, as well. Interestingly, when TPH1 expression and sera vitamin D content were plotted (Fig. 3), a pattern that differentiates between different IBS subtypes was not readily apparent. One possible explanation for these observations is that the depressed levels of TPH1 in IBSD patients may be predominantly the result of feedback repression of the TPH1 gene via elevated serotonin signaling, while lowered levels of TPH1 in IBS-C and/or IBS-M patients may be primarily the result of vitamin D insufficiency. Thus, the IBS pathological state may be correlated with dysregulated serotonin production, possibly secondary to (or in combination with) vitamin $\mathrm{D}$ deficiency. Moreover, during the administration of the current study, another clinical trial was published ${ }^{47}$ that reported an IBS cohort with appreciably depressed serum $25(\mathrm{OH}) \mathrm{D}$ levels and a significant positive association between quality of life and circulating $25(\mathrm{OH}) \mathrm{D}$ concentrations after vitamin D supplementation. Interestingly, the IBSC patients in this population were the most responsive to vitamin $\mathrm{D}$ supplementation in terms of a striking improvement across nearly all IBS symptoms. ${ }^{47}$ Because IBS-C patients are thought to possess lower gut serotonin levels, ${ }^{48}$ the responsiveness of these patients to vitamin $\mathrm{D}$ supplementation is consistent with the actions of vitamin $\mathrm{D}$ to raise gut $\mathrm{TPH} 1 /$ serotonin levels $^{25}$ and with our current hypothesis that vitamin $\mathrm{D}$, via induction of the intestinal TPH1 gene, may play a role in IBS pathophysiology.

While this study was limited by its small sample size, our IBS cohort displayed a trend toward diminished serum vitamin D levels relative to our non-IBS control group. Further, we provide preliminary evidence that vitamin D may have the capacity to alleviate some IBS symptoms via regulation of IBS-specific genes, as well as to increase expression of $\mathrm{TPH}^{25}$ to drive enhanced gut serotonin production, particularly in IBS-C and/or IBS-M patients. However, communication between the central nervous system and the gut plays a tremendous role in the modulation of GI function in a relationship termed the gut-brain axis. Furthermore, recent research has shown that GI microbiota may significantly influence the gutbrain axis through neural, endocrine, and immune signaling and may possibly contribute to certain disease states. ${ }^{49}$ Strong evidence exists that indicates the GI microbiome ${ }^{50}$ may play a role in IBS, ${ }^{51}$ that vitamin D signaling may also impact the gut microbiome, ${ }^{52}$ and that gut microbiota regulate host serotonin biosynthesis. ${ }^{53}$ Therefore, we are actively exploring interactions between vitamin $\mathrm{D}$, serotonin metabolism and related gene expression, as well as the microbiome to develop additional diagnostic modalities and to further elucidate mechanistic paradigms in IBS.

\section{Conclusion}

A total of 858 differentially expressed genes were identified, including differential expression of genes associated with serotonin metabolism. Analysis via real-time PCR revealed that IBS patientderived RNA exhibited lower levels of TPH1, the enzyme that catalyzes the rate-limiting step in serotonin biosynthesis, relative to control non-IBS samples. Moreover, values for serum 25(OH)D concentrations across both the IBS and control sample populations revealed a trend towards lower vitamin D levels within the IBS cohort, particularly in the IBS-D subtype. Finally, the expression of select IBS genetic biomarkers was shown to be modulated by vitamin $\mathrm{D}$. These results suggest that IBS pathogenesis and pathophysiology may involve dysregulated serotonin production and/or vitamin $\mathrm{D}$ insufficiency.

\section{Supplementary Materials}

Note: To access the supplementary tables mentioned in this article, visit the online version of Journal of Neurogastroenterology and Motility at http://www.jnmjournal.org/, and at https://doi. org/10.5056/jnm17021.

Acknowledgements: The authors wish to thank Donna David, Deborah Hilgemann, Deborah Johnson, Tammy Koepel, and Rachel Thiel for their contributions, as well as all the patients that participated in this study.

Financial support: This study was funded by the Marshfield Clinic Research Foundation (Grant No. AGR11/09/11) to Steven H Yale, Christopher J Rall, Todd R Sandrin, and Peter W Jurutka.

\section{Conflicts of interest: None.}

Author contributions: Steven H Yale, Todd S Sandrin, and Peter W Jurutka contributed to the concept and design of the study; Christopher M Dussik, Maryam Hockley, Aleksandra Grozić, Ichiro Kaneko, Lin Zhang, Marya S Sabir, Jin Park, Jie Wang, Cheryl A Nickerson, Amy E Foxx-Orenstein, Connie M Borror, Todd R Sandrin, and Peter W Jurutka were involved in data acquisition and analysis; Jin Park, Jie Wang, and Connie M Borror provided statistical design and support; Steven $\mathrm{H}$ Yale, Christopher J Rall, and Todd R Sandrin coordinated acquisition of data and trial 
supervision; and all authors contributed to data interpretation, critically reviewed the manuscript, and approved the final version of the manuscript.

\section{References}

1. Occhipinti K, Smith JW. Irritable bowel syndrome: a review and update. Clin Colon Rectal Surg 2012;25:46-52.

2. Chey WD, Kurlander J, Eswaran S. Irritable bowel syndrome: a clinical review. JAMA 2015;313:949-958.

3. Fass R, Fullerton S, Naliboff B, Hirsh T, Mayer EA. Sexual dysfunction in patients with irritable bowel syndrome and non-ulcer dyspepsia. Digestion 1998;59:79-85.

4. Tack J, Drossman DA. What's new in Rome IV? Neurogastroenterol Motil 2017;29.

5. Saha L. Irritable bowel syndrome: pathogenesis, diagnosis, treatment, and evidence-based medicine. World J Gastroenterol 2014;20:67596773.

6. Saito YA, Petersen GM, Larson JJ, et al. Familial aggregation of irritable bowel syndrome: a family case-control study. Am J Gastroenterol 2010;105:833-841.

7. Jostins L, Ripke S, Weersma RK, et al. Host-microbe interactions have shaped the genetic architecture of inflammatory bowel disease. Nature 2012;491:119-124.

8. Ek WE, Reznichenko A, Ripke S, et al. Exploring the genetics of irritable bowel syndrome: a GWA study in the general population and replication in multinational case-control cohorts. Gut 2015;64:1774-1782.

9. Dooley TP, Curto EV, Reddy SP, et al. Regulation of gene expression in inflammatory bowel disease and correlation with IBD drugs: screening by DNA microarrays. Inflamm Bowel Dis 2004;10:1-14.

10. Costello CM, Mah N, Häsler R, et al. Dissection of the inflammatory bowel disease transcriptome using genome-wide cDNA microarrays. PLoS Med 2005;2:e199.

11. Dieckgraefe BK, Stenson WF, Korzenik JR, Swanson PE, Harrington CA. Analysis of mucosal gene expression in inflammatory bowel disease by parallel oligonucleotide arrays. Physiol Genomics 2000;4:1-11.

12. Martínez C, Vicario M, Ramos L, et al. The jejunum of diarrheapredominant irritable bowel syndrome shows molecular alterations in the tight junction signaling pathway that are associated with mucosal pathobiology and clinical manifestations. Am J Gastroenterol 2012;107:736-746.

13. Vicario M, González-Castro AM, Martínez C, et al. Increased humoral immunity in the jejunum of diarrhoea-predominant irritable bowel syndrome associated with clinical manifestations. Gut 2015;64:1379-1388.

14. Aerssens J, Camilleri M, Talloen W, et al. Alterations in mucosal immunity identified in the colon of patients with irritable bowel syndrome. Clin Gastroenterol Hepatol 2008;6:194-205.

15. Spiller R, Lam C. An update on post-infectious irritable bowel syndrome: role of genetics, immune activation, serotonin and altered microbiome. J Neurogastroenterol Motil 2012;18:258-268.

16. Camilleri M. Serotonin in the gastrointestinal tract. Curr Opin Endocrinol Diabetes Obes 2009;16:53-59.
17. Gershon MD. 5-Hydroxytryptamine (serotonin) in the gastrointestinal tract. Curr Opin Endocrinol Diabetes Obes 2013;20:14-21.

18. Kerckhoffs AP, ter Linde JJ, Akkermans LM, Samsom M. SERT and TPH-1 mRNA expression are reduced in irritable bowel syndrome patients regardless of visceral sensitivity state in large intestine. Am J Physiol Gastrointest Liver Physiol 2012;302:G1053-G1060.

19. Coates MD, Mahoney CR, Linden DR, et al. Molecular defects in mucosal serotonin content and decreased serotonin reuptake transporter in ulcerative colitis and irritable bowel syndrome. Gastroenterology 2004;126:1657-1664

20. Dunlop SP, Coleman NS, Blackshaw E, et al. Abnormalities of 5-hydroxytryptamine metabolism in irritable bowel syndrome. Clin Gastroenterol Hepatol 2005;3:349-357.

21. Ford AC, Quigley EM, Lacy BE, et al. Effect of antidepressants and psychological therapies, including hypnotherapy, in irritable bowel syndrome: systematic review and meta-analysis. Am J Gastroenterol 2014;109:1350-1365.

22. Haussler MR, Whitfield GK, Haussler CA, Hsieh JC, Jurutka PW. Nuclear vitamin D receptor: natural ligands, molecular structure-function, and transcriptional control of vital genes. In: Feldman D, Pike JW, Adams J, eds. Vitamin D. 3 ed. San Diego: Academic Press 2011:137170 .

23. Nwosu BU, Maranda L, Candela N. Vitamin D status in pediatric irritable bowel syndrome. PLoS One 2017;12:e0172183.

24. Cantorna MT, McDaniel K, Bora S, Chen J, James J. Vitamin D, immune regulation, the microbiota, and inflammatory bowel disease. Exp Biol Med (Maywood) 2014;239:1524-1530.

25. Kaneko I, Sabir MS, Dussik CM, et al. 1,25-Dihydroxyvitamin D regulates expression of the tryptophan hydroxylase 2 and leptin genes: implication for behavioral influences of vitamin D. FASEB J 2015;29:40234035.

26. Ritchie ME, Phipson B, Wu D, et al. limma powers differential expression analyses for RNA-sequencing and microarray studies. Nucleic Acids Res 2015;43:e47.

27. Bindea G, Mlecnik B, Hackl H, et al. ClueGO: a cytoscape plug-in to decipher functionally grouped gene ontology and pathway annotation networks. Bioinformatics 2009;25:1091-1093.

28. Wu G, Dawson E, Duong A, Haw R, Stein L. ReactomeFIViz: a cytoscape app for pathway and network-based data analysis. F1000Res 2014;3:146.

29. Shannon P, Markiel A, Ozier O, et al. Cytoscape: a software environment for integrated models of biomolecular interaction networks. Genome Res 2003;13:2498-2504.

30. Hollis BW. Quantitation of 25-hydroxyvitamin D and 1,25-dihydroxyvitamin $\mathrm{D}$ by radioimmunoassay using radioiodinated tracers. Methods Enzymol 1997;282:174-186.

31. Holick MF, Binkley NC, Bischoff-Ferrari HA, et al. Evaluation, treatment, and prevention of vitamin D deficiency: an endocrine society clinical practice guideline. J Clin Endocrinol Metab 2011;96:1911-1930.

32. Holick MF. Vitamin D deficiency. N Engl J Med 2007;357:266-281.

33. Witmer AN, van Blijswijk BC, Dai J, et al. VEGFR-3 in adult angiogenesis. J Pathol 2001;195:490-497. 
34. Zhou R. The Eph family receptors and ligands. Pharmacol Ther 1998;77:151-181.

35. Akpinar M, Lesche M, Fanourgakis G, et al. TDRD6 mediates early steps of spliceosome maturation in primary spermatocytes. PLoS Genet 2017;13:e1006660.

36. Gershon MD, Tack J. The serotonin signaling system: from basic understanding to drug development for functional GI disorders. Gastroenterology 2007;132:397-414.

37. Grasberger H, Chang L, Shih W, et al. Identification of a functional TPH1 polymorphism associated with irritable bowel syndrome bowel habit subtypes. Am J Gastroenterol 2013;108:1766-1774.

38. Abbasnezhad A, Amani R, Hajiani E, Alavinejad P, Cheraghian B, Ghadiri A. Effect of vitamin D on gastrointestinal symptoms and healthrelated quality of life in irritable bowel syndrome patients: a randomized double-blind clinical trial. Neurogastroenterol Motil 2016;28:1533-1544.

39. Khayyat Y, Attar S. Vitamin D deficiency in patients with irritable bowel syndrome: does it exist? Oman Med J 2015;30:115-118.

40. Haussler MR, Whitfield GK, Haussler CA, et al. 1,25-dihydroxyvitamin $\mathrm{D}$ and klotho: a tale of two renal hormones coming of age. Vitam Horm 2016;100:165-230.

41. Dussik CM, Grozić A, Hockley M, et al. Characterization of vitamin $\mathrm{D}$ and serotonin pathway variations in patients with irritable bowel syndrome. The FASEB J 2016;30(suppl 1):828.2.

42. Haussler MR, Whitfield GK, Haussler CA, et al. The nuclear vitamin $\mathrm{D}$ receptor: biological and molecular regulatory properties revealed. J Bone Miner Res 1998;13:325-349.

43. Nozawa K, Kawabata-Shoda E, Doihara H, et al. TRPA1 regulates gastrointestinal motility through serotonin release from enterochromaffin cells. Proc Natl Acad Sci USA 2009;106:3408-3413.

44. Gershon MD. Review article: serotonin receptors and transporters -- roles in normal and abnormal gastrointestinal motility. Aliment Pharmacol Ther 2004;20(suppl 7):3-14.

45. Chen JJ, Li Z, Pan H, et al. Maintenance of serotonin in the intestinal mucosa and ganglia of mice that lack the high-affinity serotonin transporter: abnormal intestinal motility and the expression of cation transporters. J Neurosci 2001;21:6348-6361.

46. Sabir MS, Dussik CM, Kaneko I, Whitfield GK, Haussler MR, Jurutka $\mathrm{PW}$. Modulation of serotonin synthesis, reuptake, and degradation by 1,25-dihydroxyvitamin $\mathrm{D}$ : potential relevance for neuropsychiatric disorders. The FASEB J 2016;30(suppl 1):869.4.

47. Tazzyman S, Richards N, Trueman AR, et al. Vitamin D associates with improved quality of life in participants with irritable bowel syndrome: outcomes from a pilot trial. BMJ Open Gastroenterol 2015;2:e000052.

48. De Ponti F. Pharmacology of serotonin: what a clinician should know. Gut 2004;53:1520-1535.

49. Cryan JF, Dinan TG. Mind-altering microorganisms: the impact of the gut microbiota on brain and behaviour. Nat Rev Neurosci 2012;13:701712 .

50. Kinross JM, von Roon AC, Holmes E, Darzi A, Nicholson JK. The human gut microbiome: implications for future health care. Curr Gastroenterol Rep 2008;10:396-403.

51. Kennedy PJ, Cryan JF, Dinan TG, Clarke G. Irritable bowel syndrome: a microbiome-gut-brain axis disorder? World J Gastroenterol 2014;20:14105-14125.

52. Dougherty U, Mustafi R, Wang Y, et al. 79 Vitamin D receptor inactivation alters the colonic microbiome and increases secondary bile acids and colonic tumorigenesis. Gastroenterology 2013;144:S-17.

53. Yano JM, Yu K, Donaldson GP, et al. Indigenous bacteria from the gut microbiota regulate host serotonin biosynthesis. Cell 2015;161:264-276. 